\title{
Biologically inspired topology optimization model with a local density penalization *
}

\author{
Misaki Sakashita* Tsuguo Kondoh** Atsushi Kawamoto** \\ Emmanuel Tromme** Shigeru Kondo* \\ * Osaka University, Suita, Osaka 565-0871, Japan \\ (e-mail: sakashitamsk@gmail.com) \\ ** Toyota Central RED Labs., Inc., Nagakute, Aichi 480-1192, Japan \\ (e-mail: atskwmt@mosk.tytlabs.co.jp)
}

Keywords: Topology Optimization, Wolff's law, Vertebra, Local density penalization

\section{INTRODUCTION}

Wolff's law states that bone morphology evolves according to their external mechanical loading. Following this law, researchers have tried to simulate bone shape formation, especially for trabecular bone, using topology optimization (Huiskes et al., 2000). Less attention has been given to the bone outer shape, composed of cortical bone. However, trabecular bone and cortical bone are both mainly formed by osteoblasts and osteoclasts. Therefore, we hypothesize that the bone outer shape also adapts to the external forces. The aim of this research is to understand the mechanism that generates the bone outer shape by reproducing the latter using topology optimization.

The mathematical model we developed is inspired by the fish vertebra. The fish vertebra can be divided into two parts (Fig. 1): an inner hourglass-like structure and an outer lateral structure (blue-colored part). Based on our observations, it turns out that numerous species present a similar hourglass-like structure but that the lateral structure strongly depends on the fish species (Fig. 2). Lateral structures can be classified into two types. The first type exhibits a ridge structure with one or more thick bone plates and the second type exhibits a network structure in which fine bones are randomly oriented. These differences seem related to the fish motion, i.e. the swimming type of the fish, and therefore, it is assumed that lateral structures also evolve based on external loading conditions. Because fish has the variety of swimming type, the model we built can produce the structure resistant to the various movements respectively as well as explain the bone formation.

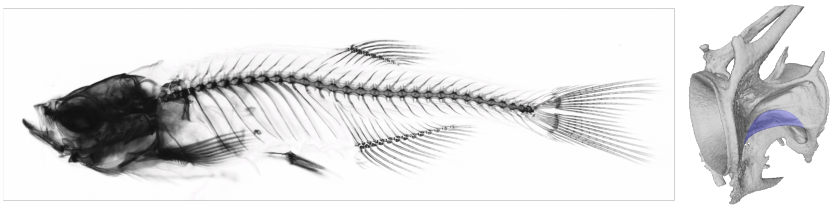

Fig. 1. Zebrafish skeleton (left) and a single vertebra scanned with micro-CT (right). The zebrafish is a model organism.

\footnotetext{
* The authors acknowledge CREST and Toyota Central R\&D Labs for their support.
}

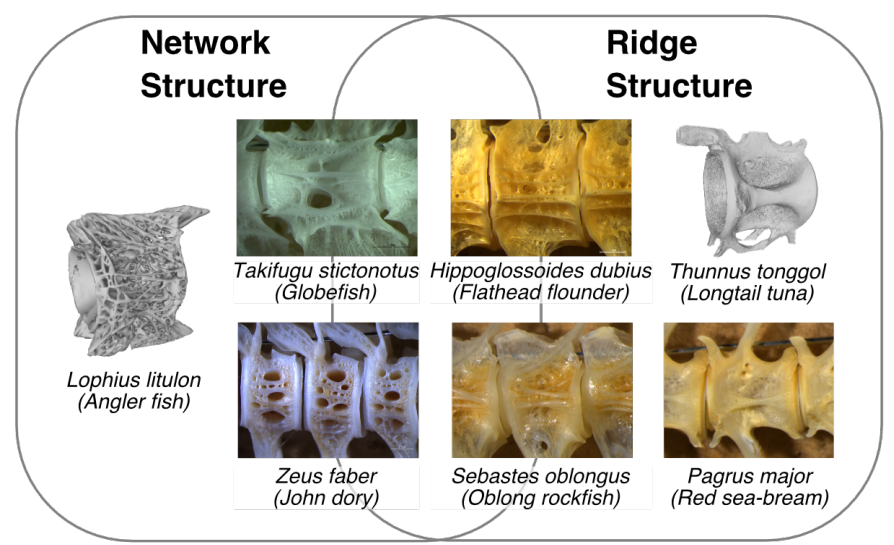

Fig. 2. Varieties of fish vertebra shape.

In standard topology optimization, the density at each material point is only constrained by the total volume of material. However, the activity of osteoblast and osteoclast is more a local phenomenon. Hence, the standard topology optimization problem is supplemented with a local density penalization to mimic this local phenomenon. The developed mathematical model to study the fish vertebra formation is hereafter detailed.

\section{MATHEMATICAL MODELING}

The adopted optimization problem governing the bone density $\rho=\rho(\boldsymbol{x})$ distribution is stated as

$$
\begin{aligned}
& \min _{\rho(\boldsymbol{x})} f[\rho] \equiv f_{0}[\rho]+f_{\text {stab }}[\rho]+f_{\text {local }}[\bar{\rho}[\rho]] \\
& \text { s.t. } g[\rho] \equiv \int_{\Omega} \rho d \Omega-\bar{V} \leq 0
\end{aligned}
$$

where $\Omega$ is the design domain and $\bar{V}$ the upper bound of the total volume constraint $g[\rho]$. The objective function $f[\rho]$ is composed of three functions that read,

$$
\begin{aligned}
f_{0}[\rho] & \equiv \int_{\Omega} \tilde{\sigma}[\rho]: \tilde{\varepsilon}[\rho] d \Omega \\
f_{\text {stab }}[\rho] & \equiv C_{\text {stab }} \int_{\Omega}(\nabla \rho)^{2} d \Omega, C_{\text {stab }}>0 \\
f_{\text {local }}[\bar{\rho}[\rho]] & \equiv C_{\text {local }} \int_{\Omega} \mathrm{H}\left(\bar{\rho}[\rho]-\bar{\rho}_{\text {local }}\right) d \Omega, C_{\text {local }}>0 .
\end{aligned}
$$


$f_{0}[\rho]$ is the total strain energy in the design domain $(\tilde{\sigma}$ and $\tilde{\varepsilon}$ are the stress and strain tensors $). f_{\text {stab }}[\rho]$ is a diffusion term smoothing the distribution of $\rho . f_{\text {local }}[\bar{\rho}[\rho]]$ enforces a penalty to the objective function when the locally averaged density $\bar{\rho}$ exceeds the prescribed upper bound $\bar{\rho}_{\text {local }}(\mathrm{H}$ is the Heaviside function). $\bar{\rho}$ is obtained by averaging $\rho=\rho(\boldsymbol{x})$ with the Helmholtz PDE based filter (Kawamoto et al., 2011) as:

$$
\begin{array}{r}
\bar{\rho}=\bar{\rho}(\boldsymbol{x}): \quad-R^{2} \nabla^{2} \bar{\rho}+\bar{\rho}=\rho \quad \text { in } \Omega, \\
R^{2} \frac{\partial \bar{\rho}}{\partial n}=0 \quad \text { on } \quad \Gamma \equiv \partial \Omega ; \quad R>0,
\end{array}
$$

where $R$ is the filtering radius.

To solve the optimization problem (1), a method based on a time dependent reaction-diffusion equation (6) is employed (Kawamoto et al., 2013). Equation (6) is driven by the sensitivity $S$, in which the Lagrangian multiplier $\lambda$ is introduced as follows:

$$
\begin{array}{r}
\rho=\rho(\boldsymbol{x}, t): \quad m \frac{\partial \rho}{\partial t}=-S \quad \text { in } \Omega, \\
C_{\text {stab }} \frac{\partial \rho}{\partial n}=0 \quad \text { on } \quad \Gamma ; \\
m>0, \quad S \equiv \frac{\delta F[\rho]}{\delta \rho}, \quad F[\rho] \equiv f[\rho]+\lambda g[\rho] .
\end{array}
$$

Solving this optimization problem gives an optimized structure with respect to the imposed boundary and loading conditions. The penalization term enables to control locally the feature size.

\section{NUMERICAL EXAMPLES}

The proposed mathematical model is implemented in the commercial software COMSOL Multiphysics ${ }^{\circledR}$. The mathematics module as well as the solid mechanics module are used.

A 2-D cantilever beam problem is first solved to demonstrate the developed method. The results illustrate that finer features can be obtained by increasing the penalization on local densities (Fig. 3).

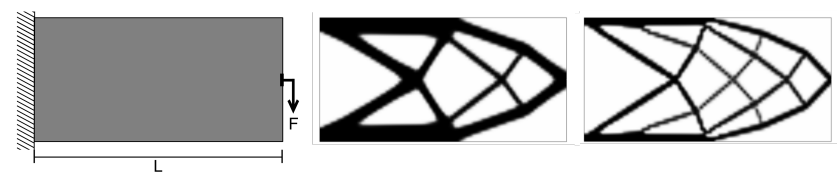

Fig. 3. Cantilever beam design problem (left) and the results with small effect (center) and large effect (right) of local density penalization.

The 3-D design domain of the fish vertebra is illustrated in Fig. 4. Since the hourglass-like structure is assumed to be identical for all fish species, the part of the design domain illustrated in Fig. 4 (on the left) is composed of bones $(\rho=1)$ regardless of external forces. The yellow parts, composed of two wedges of $30^{\circ}$ spaced by $180^{\circ}$, contain nerves and blood vessels, thus no bone is allowed $(\rho=0)$. Finally, the design domain is thus composed of the two external blue parts. Compression forces are applied to the external surface of the hourglass structure (red-colored surfaces) in the axial direction (black arrows).
Without local density penalization, thick beams appear similarly to the ridge structure. Penalizing locally the density, thinner beams are promoted and they tend to form a network (Fig. 5).
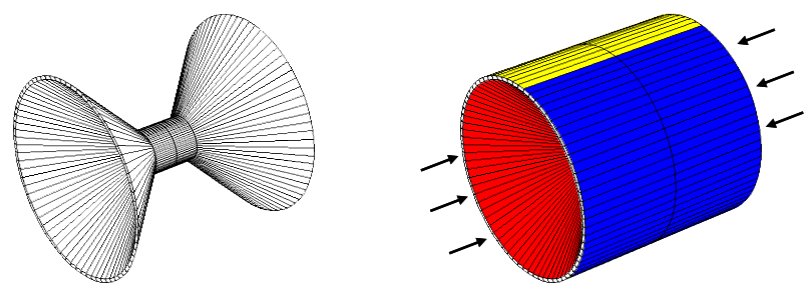

Fig. 4. Design domain of the fish vertebra.
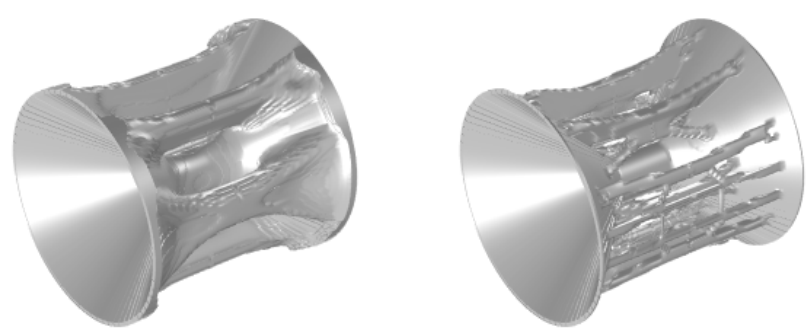

Fig. 5. Optimization result without local density penalization (left) and with local density penalization (right).

\section{CONCLUSION}

A mathematical model has been developed to generate fish vertebra using topology optimization combined with a local density penalization. Numerical results show that the proposed model can produce both types of lateral structures, i.e. ridge structure and network structure, which are similar to the actual fish bone. In the future, it would be interesting to be able to produce various forms of fish vertebra by only adjusting a few parameters of the penalization law.

\section{REFERENCES}

Huiskes, R., Ruimerman, R., van Lenthe, G.H., and Janssen, J.D. (2000). Effects of mechanical forces on maintenance and adaptation of form in trabecular bone. Nature, 405(6787), 704-706. doi:10.1038/35015116.

Kawamoto, A., Matsumori, T., Nomura, T., Kondoh, T., Yamasaki, S., and Nishiwaki, S. (2013). Topology optimization by a time-dependent diffusion equation. International Journal for Numerical Methods in Engineering, 93(8), 795-817. doi:10.1002/nme.4407.

Kawamoto, A., Matsumori, T., Yamasaki, S., Nomura, T., Kondoh, T., and Nishiwaki, S. (2011). Heaviside projection based topology optimization by a PDE-filtered scalar function. Structural and Multidisciplinary Optimization, 44(1), 19-24. doi:10.1007/s00158-010-0562-2. 